\title{
On Hilbert's Integral Inequality and Its Applications
}

\author{
Waad T. Sulaiman \\ Department of Computer Engineering, College of Engineering, University of Mosul, Mosul, Iraq. \\ E-mail: waadsulaiman@hotmail.com
}

Received December 3, 2010; revised January 29, 2011; accepted February 2, 2011

\section{Abstract}

The main result of this paper is presented as follows Let $f, g, h, k \geq 0$, $h$ is homogeneous and symmetric of degree $\lambda$ and $F(x, y)=1-k(x)+k(y) \geq 0$. Then

$$
\begin{aligned}
& \left(\int_{0}^{\infty} \int_{0}^{\infty} \frac{f(x) g(y)}{h(x, y)} d x d y\right)^{4} \leq\left(\left(C \int_{0}^{\infty} x^{1-\lambda} f^{2}(x) d x\right)^{2}-\left(\int_{0}^{\infty}(C k(x)-C(x)) x^{1-\lambda} f^{2}(x) d x\right)^{2}\right) \\
& \times\left(\left(C \int_{0}^{\infty} x^{1-\lambda} g^{2}(x) d x\right)^{2}-\left(\int_{0}^{\infty}(C k(x)-C(x)) x^{1-\lambda} g^{2}(x) d x\right)^{2}\right),
\end{aligned}
$$

where

$$
C=\int_{0}^{\infty} \frac{t^{a}}{h(1, t)} d t, \quad C(x)=\int_{0}^{\infty} \frac{k(x t) t^{a}}{h(1, t)} d t,
$$

provided the integrals on the RHS do exists. Some other special cases are also deduced.

Keywords: Hilbert's Integral Inequality, Holder's Inequality, Weight Function

\section{Introduction}

If

$$
\begin{aligned}
& f, g \geq 0, \frac{1}{p}+\frac{1}{q}=1, \\
& 0<\int_{0}^{\infty} f^{p}(x) d x<\infty, 0<\int_{0}^{\infty} g^{q}(x) d x<\infty,
\end{aligned}
$$

then

$$
\begin{aligned}
& \int_{0}^{\infty} \int_{0}^{\infty} \frac{f(x) g(y)}{x+y} d x d y \\
& \leq \frac{\pi}{\sin (\pi / p)}\left(\int_{0}^{\infty} f^{p}(x) d x\right)^{1 / p}\left(\int_{0}^{\infty} g^{q}(x) d x\right)^{1 / q}
\end{aligned}
$$

where the constant factor $\pi / \sin (\pi / p)$ is the best possible. Many mathematicians presented generalizations or new kinds of (1). Hardy inequality is very important in analysis theory and applications, it has been absorbing much interest of analysis see $([1,2])$.Very recently P. X. Ying and G. Mingzhe (see [3]) proved the following new kind
Theorem 1.1. Let $f(x)$ be a real function. If $0<\int_{0}^{\infty} f^{2}(x) d x<\infty$, then

$$
\begin{aligned}
& \left(\int_{0}^{\infty} \int_{0}^{\infty} \frac{f(x) f(y)}{x+y} d x d y\right)^{2} \\
& <\pi^{2}\left(\left(\int_{0}^{\infty} f^{p}(x) d x\right)^{2}-\left(\int_{0}^{\infty} \varpi(x) f^{p}(x) d x\right)^{2}\right)
\end{aligned}
$$

where $\varpi(x)=\frac{1}{1+\sqrt{x}}-\frac{1}{1+x}$.

\section{Lemma}

The following lemma is needed for our aim.

Lemma 2.1. Let $h(x, y)$ be symmetric. Then

$$
\int_{0}^{\infty} \int_{0}^{\infty} \frac{f(x) f(y)}{h(x, y)} F(x, y) d x d y=\int_{0}^{\infty} \int_{0}^{\infty} \frac{f(x) f(y)}{h(x, y)} d x d y
$$

where $F(x, y)=1-k(x)+k(y)$

\section{Proof.}




$$
\begin{aligned}
& \int_{0}^{\infty} \int_{0}^{\infty} \frac{f(x) f(y)}{h(x, y)} F(x, y) d x d y=\int_{0}^{\infty} \int_{0}^{\infty} \frac{f(x) f(y)}{h(x, y)}(1-k(x)+k(y)) d x d y \\
& =\int_{0}^{\infty} \int_{0}^{\infty} \frac{f(x) f(y)}{h(x, y)} d x d y-\int_{0}^{\infty} \int_{0}^{\infty} \frac{f(x) f(y) k(x)}{h(x, y)} d x d y+\int_{0}^{\infty} \int_{0}^{\infty} \frac{f(x) f(y) k(y)}{h(x, y)} d x d y \\
& =\int_{0}^{\infty} \int_{0}^{\infty} \frac{f(x) f(y)}{h(x, y)} d x d y-\int_{0}^{\infty} \int_{0}^{\infty} \frac{f(x) f(y) k(x)}{h(x, y)} d x d y+\int_{0}^{\infty} \int_{0}^{\infty} \frac{f(y) f(x) k(x)}{h(y, x)} d y d x \\
& =\int_{0}^{\infty} \int_{0}^{\infty} \frac{f(x) f(y)}{h(x, y)} d x d y-\int_{0}^{\infty} \int_{0}^{\infty} \frac{f(x) f(y) k(x)}{h(x, y)} d x d y+\int_{0}^{\infty} \int_{0}^{\infty} \frac{f(x) f(y) k(x)}{h(x, y)} d x d y=\int_{0}^{\infty} \int_{0}^{\infty} \frac{f(x) f(y)}{h(x, y)} d x d y
\end{aligned}
$$

The object of this paper is to present the following general result

\section{Main Results}

Theorem 3.1. Let $f, g, h, k \geq 0, h$ is homogeneous and

$$
\begin{aligned}
\left(\int_{0}^{\infty} \int_{0}^{\infty} \frac{f(x) g(y)}{h(x, y)} d x d y\right)^{4} \leq & \left(\left(C \int_{0}^{\infty} x^{1-\lambda} f^{2}(x) d x\right)^{2}-\left(\int_{0}^{\infty}(C k(x)-C(x)) x^{1-\lambda} f^{2}(x) d x\right)^{2}\right) \\
& \times\left(\left(C \int_{0}^{\infty} x^{1-\lambda} g^{2}(x) d x\right)^{2}-\left(\int_{0}^{\infty}(C k(x)-C(x)) x^{1-\lambda} g^{2}(x) d x\right)^{2}\right),
\end{aligned}
$$

where

$$
C=\int_{0}^{\infty} \frac{t^{a}}{h(1, t)} d t, \quad C(x)=\int_{0}^{\infty} \frac{k(x t) t^{a}}{h(1, t)} d t,
$$

provided the integrals on the RHS do exists.

\section{Proof.}

$$
\begin{gathered}
\int_{0}^{\infty} \int_{0}^{\infty} \frac{f(x) g(y) F(x, y)}{h(x, y)} d x d y=\int_{0}^{\infty} \int_{0}^{\infty} \frac{f(x) \sqrt{F(x, y)}}{\sqrt{h(x, y)}} \times \frac{g(y) \sqrt{F(x, y)}}{\sqrt{h(x, y)}} d x d y \\
\leq\left(\int_{0}^{\infty} \int_{0}^{\infty} \frac{f^{2}(x) F(x, y)}{h(x, y)} d x d y\right)^{1 / 2}\left(\int_{0}^{\infty} \int_{0}^{\infty} \frac{g^{2}(y) F(x, y)}{h(x, y)} d x d y\right)^{1 / 2}=\sqrt{M} \sqrt{N} . \\
M=\int_{0}^{\infty} \int_{0}^{\infty} \frac{f(x) \sqrt{F(x, y)}}{\sqrt{h(x, y)}}\left(\frac{y}{x}\right)^{a / 2} \times \frac{f(x) \sqrt{F(x, y)}\left(\frac{x}{y}\right)^{a / 2} d x d y}{\sqrt{h(x, y)}} \\
\leq\left(\int_{0}^{\infty} \int_{0}^{\infty} \frac{f^{2}(x) F(x, y)}{h(x, y)}\left(\frac{y}{x}\right)^{a} d x d y\right)^{1 / 2}\left(\int_{0}^{\infty} \int_{0}^{\infty} \frac{f^{2}(x) F(x, y)}{h(x, y)}\left(\frac{y}{x}\right)^{a} d x d y\right)^{1 / 2}=\sqrt{M_{1}} \sqrt{M_{2}} . \\
M_{1}=\int_{0}^{\infty} \int_{0}^{\infty} \frac{f^{2}(x)(1-k(x)+k(y))}{h(x, y)}\left(\frac{y}{x}\right)^{a} d x d y=\int_{0}^{\infty} f^{2}(x)(1-k(x)) \int_{0}^{\infty} \frac{(y / x)^{a}}{h(x, y)} d y d x+\int_{0}^{\infty} f^{2}(x) \int_{0}^{\infty} \frac{k(y)(y / x)^{a}}{h(x, y)} d y d x \\
=\int_{0}^{\infty} x^{1-\lambda}(1-k(x)) f^{2}(x) \int_{0}^{\infty} \frac{u^{a}}{h(1, u)} d u d x+\int_{0}^{\infty} x^{1-\lambda} f^{2}(x) \int_{0}^{\infty} \frac{u^{a} k(x u)}{h(1, u)} d u d x \\
=C \int_{0}^{\infty} x^{1-\lambda} f^{2}(x) d x-\int_{0}^{\infty}(C k(x)-C(x)) x^{1-\lambda} f^{2}(x) d x .
\end{gathered}
$$


Similarly,

$M_{2}=C \int_{0}^{\infty} x^{1-\lambda} f^{2}(x) d x+\int_{0}^{\infty}(C k(x)-C(x)) x^{1-\lambda} f^{2}(x) d x$.

Therefore

$$
\begin{aligned}
M^{2}=\left(C \int_{0}^{\infty} x^{1-\lambda} f^{2}(x) d x\right)^{2} & -\left(\int_{0}^{\infty}(C k(x)-C(x))\right. \\
& -\left(\int_{0}^{\infty}(C k(x)-C(x)) x^{1-\lambda} f^{2}(x) d x\right)^{2}, \quad \text { Applying lemma } 2.1 \text { to have } \\
& \left(\int_{0}^{\infty} \int_{0}^{\infty} \frac{f(x) g(y)}{h(x, y)} d x d y\right)^{4}=\left(\int_{0}^{\infty} \int_{0}^{\infty} \frac{f(x) g(y) F(x, y)}{h(x, y)} d x d y\right)^{4} \\
& \leq\left(\left(C \int_{0}^{\infty} x^{1-\lambda} f^{2}(x) d x\right)^{2}-\left(\int_{0}^{\infty}(C k(x)-C(x)) x^{1-\lambda} f^{2}(x) d x\right)^{2}\right) \\
& \times\left(\left(C \int_{0}^{\infty} x^{1-\lambda} g^{2}(x) d x\right)^{2}-\left(\int_{0}^{\infty}(C k(x)-C(x)) x^{1-\lambda} g^{2}(x) d x\right)^{2}\right)
\end{aligned}
$$

Remark. It may be mentioned that theorem 1.1 follows from corollary 4.1 by putting $h(x, y)=x+y, \quad a=-1 / 2, \quad \lambda=1, \quad k(x)=1 /(1+x)$ as follows

$$
\begin{array}{cr}
C=\int_{0}^{\infty} \frac{u^{-1 / 2}}{1+u} d u=2 \int_{0}^{\infty} \frac{d u}{1+u^{2}}=\pi . & C k(x)-C(x)=\pi\left(\frac{1}{1+x}-\frac{1}{1+\sqrt{x}}\right) \\
C(x)=\int_{0}^{\infty} \frac{k(x u) u^{-1 / 2}}{1+u} d u=\int_{0}^{\infty} \frac{k\left(x u^{2}\right)}{1+u^{2}} d u & \text { Theorem 3.2. Let } f \geq 0,0 \leq g \leq 1, \lambda>0 . \text { Then } \\
\left(\int_{0}^{\infty} \int_{0}^{\infty} \frac{f(x) f(y) \sqrt{1-g(x)} \sqrt{1+g(y)}}{(x+y)^{\lambda}} d x d y\right)^{2} \leq B^{2}\left(\frac{\lambda}{2}, \frac{\lambda}{2}\right)\left(\left(\int_{0}^{\infty} x^{1-\lambda} f^{2}(x) d x\right)^{2}-\left(\int_{0}^{\infty} x^{1-\lambda} f^{2}(x) g(x) d x\right)^{2}\right) .
\end{array}
$$

and

\section{Proof.}

$$
\begin{aligned}
& \int_{0}^{\infty} \int_{0}^{\infty} \frac{f(x) f(y) \sqrt{1-g(x)} \sqrt{1+g(y)}}{(x+y)^{\lambda}} d x d y=\int_{0}^{\infty} \int_{0}^{\infty} \frac{f(x) \sqrt{1-g(x)}}{(x+y)^{\lambda / 2}}\left(\frac{y}{x}\right)^{\frac{\lambda}{4}-\frac{1}{2}} \times \frac{f(y) \sqrt{1+g(y)}}{(x+y)^{\lambda / 2}}\left(\frac{x}{y}\right)^{\frac{\lambda}{4}-\frac{1}{2}} d x d y \\
& \leq\left(\int_{0}^{\infty} \int_{0}^{\infty} \frac{f^{2}(x)(1-g(x))}{(x+y)^{\lambda}}\left(\frac{y}{x}\right)^{\frac{\lambda}{2}-1} d x d y\right)^{1 / 2}\left(\int_{0}^{\infty} \int_{0}^{\infty} \frac{f^{2}(y)(1+g(y))}{(x+y)^{\lambda}}\left(\frac{x}{y}\right)^{\frac{\lambda}{2}-1} d x d y\right)^{1 / 2}=\sqrt{G} \sqrt{H} . \\
& G=\int_{0}^{\infty} f^{2}(x)(1-g(x))\left(\int_{0}^{\infty} \frac{(y / x)^{\frac{\lambda}{2}-1}}{(x+y)^{\lambda}} d y\right) d x=\int_{0}^{\infty} f^{2}(x)(1-g(x))\left(\int_{0}^{\infty} \frac{x^{1-\lambda} t^{\frac{\lambda}{2}-1}}{(1+t)^{\lambda}} d t\right) d x=B\left(\frac{\lambda}{2}, \frac{\lambda}{2}\right) \int_{0}^{\infty} x^{1-\lambda} f^{2}(x)(1-g(x)) d x
\end{aligned}
$$

Similarly 


$$
H=B\left(\frac{\lambda}{2}, \frac{\lambda}{2}\right) \int_{0}^{\infty} y^{1-\lambda} f^{2}(y)(1+g(y)) d y,
$$

and hence

$$
\left(\int_{0}^{\infty} \int_{0}^{\infty} \frac{f(x) f(y) \sqrt{1-g(x)} \sqrt{1+g(y)}}{(x+y)^{\lambda}} d x d y\right)^{2} \leq G H=B^{2}\left(\frac{\lambda}{2}, \frac{\lambda}{2}\right)\left(\left(\int_{0}^{\infty} x^{1-\lambda} f^{2}(x) d x\right)^{2}-\left(\int_{0}^{\infty} x^{1-\lambda} f^{2}(x) g(x) d x\right)^{2}\right) .
$$

\section{Applications}

Corollary 4.1. Let $f, h, k \geq 0, h$ is homogeneous and symmetric of degree $\lambda$.

Then

$$
\left(\int_{0}^{\infty} \int_{0}^{\infty} \frac{f(x) f(y)}{h(x, y)} d x d y\right)^{2} \leq\left(\left(C \int_{0}^{\infty} x^{1-\lambda} f^{2}(x) d x\right)^{2}-\left(\int_{0}^{\infty}(C k(x)-C(x)) x^{1-\lambda} f^{2}(x) d x\right)^{2}\right)
$$

where

$$
C=\int_{0}^{\infty} \frac{t^{a}}{h(1, t)} d t, \quad C(x)=\int_{0}^{\infty} \frac{k(x t) t^{a}}{h(1, t)} d t,
$$

provided the integrals on the RHS do exists.
Proof. The proof follows from theorem 3.1 by putting $g=f$

Corollary 4.2. Let $f, h, k \geq 0, \mathrm{~h}$ is homogeneous and symmetric of degree $\lambda$.

Then

$$
\left(\int_{0}^{\infty} \int_{0}^{\infty} \frac{f(x) f(y)}{(x+y)^{\lambda}} d x d y\right)^{2} \leq\left(\left(C \int_{0}^{\infty} x^{1-\lambda} f^{2}(x) d x\right)^{2}-\left(\int_{0}^{\infty}(C k(x)-C(x)) x^{1-\lambda} f^{2}(x) d x\right)^{2}\right)
$$

where

$$
C=\int_{0}^{\infty} \frac{t^{a}}{(1+t)^{\lambda}} d t, \quad C(x)=\int_{0}^{\infty} \frac{k(x t) t^{a}}{(1+t)^{\lambda}} d t,
$$

provided the integrals on the RHS do exists.
Proof. The proof follows from corollary 4.1 by putting $h(x, y)=(x+y)^{\lambda}$.

Corollary 4.3. Let $f, h, k \geq 0, h$ is homogeneous and symmetric of degree $\lambda$.

Then

$$
\left(\int_{0}^{\infty} \int_{0}^{\infty} \frac{f(x) f(y)}{x^{\lambda}+y^{\lambda}} d x d y\right)^{2} \leq\left(\left(C \int_{0}^{\infty} x^{1-\lambda} f^{2}(x) d x\right)^{2}-\left(\int_{0}^{\infty}(C k(x)-C(x)) x^{1-\lambda} f^{2}(x) d x\right)^{2}\right)
$$

where

$$
C=\int_{0}^{\infty} \frac{t^{a}}{1+t^{\lambda}} d t, \quad C(x)=\int_{0}^{\infty} \frac{k(x t) t^{a}}{1+t^{\lambda}} d t,
$$

provided the integrals on the RHS do exists.

Proof. The proof follows from corollary 4.1 by putting $h(x, y)=x^{\lambda}+y^{\lambda}$.

\section{References}

[1] G. H. Hardy, J. E. Littlewood and G. Polya, "Inequali- ties,” Cambridge University Press, Cambridge, 1952.

[2] J. E. Mitronovic, J. E. Pecaric and A. M. Fink, "Inequalities Involving Functions and Their Integrals and Derivatives,” Boston Kluwer Academic Publishers, 1991.

[3] P. X. Ying and G. Mingzhe, "On Hilbert's Integral Inequality and Its Applications,” Journal of Applied Mathematics, Preprint. 